\title{
Multiple Constrained Optimal Experimental Design ${ }^{1)}$
}

\author{
Myung-Wook Kahng2), Young II Kim ${ }^{3)}$
}

\begin{abstract}
It is unpractical for the optimal design theory based on the given model and assumption to be applied to the real-world experimentation. Particularly, when the experimenter feels it necessary to consider multiple objectives in experimentation, its modified version of optimality criteria is indeed desired. The constrained optimal design is one of many methods developed in this context. But when the number of constraints exceeds two, there always exists a problem in specifying the lower limit for the efficiencies of the constraints because the "infeasible solution" issue arises very quickly. In this paper, we developed a sequential approach to tackle this problem assuming that all the constraints can be ranked in terms of importance. This approach has been applied to the polynomial regression model.
\end{abstract}

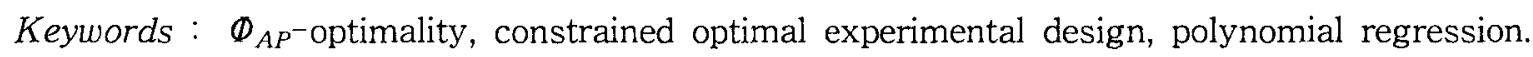

\section{1. 소 개}

실험자는 반응변수 $y$ 와 설명인자 $x$ 와의 관계를 설정하기 위하여 다음과 같은 다항회귀모형 (polynomial regression model)을 염두에 둔다고 하자.

$$
y=\theta_{0}+\sum_{i=1}^{k} \theta_{i} x^{i}+\varepsilon=f_{k}(x)^{T} \theta+\varepsilon
$$

여기서 $y$ 는 관측 가능한 반응변수이며 $\theta=\left(\theta_{0}, \cdots, \theta_{k}\right)^{T}$ 는 $(k+1) \times 1$ 의 알려지지 않은 모수벡 터이며 $\varepsilon$ 는 서로 비상관인 실수값을 가지는 기대값이 0 이고 분산이 $\sigma^{2}$ 인 오차확률변수이다. 앞 으로는 일반성의 손실 없이 $\sigma^{2}$ 은 1 로 가정한다. 실험계획문제는 실험영역 $\Omega=[-1,1]$ 안에 있 는 $s$ 개 점의 유한집합에 질량을 부여하는 확률질량함수 $\xi$ 로 기술될 수 있다. 표본크기 $n$ 이 적 은 실험계획에서는 $n \xi\left(x_{i}\right), i=1, \cdots, s$ 가 정수이어야 하는 제약을 지니지만 본 연구에서는 이러 한 제약조건이 부여되지 않는 표본이 큰 경우만을 고려한다. 참고로 $x_{i}$ 는 실험계획 $\xi$ 의 받침점

1) Kahng's research was supported by the Sookmyung Women's University Research Grants 2001, and Kim's research was supported by the 2001 Chungang University Research Fund.

2) Professor, Department of Statistics, Sookmyung Women's University, Seoul, 140-742 KOREA E-mail: mwkahng@sookmyung.ac.kr

3) Professor, Department of Information System, Chungang University, Kyunggi-Do, 456-756 KOREA E-mail: yik01@cau.ac.kr 
(supporting point)이라 부른다.

실험계획 $\xi$ 에 내재되어 있는 정보에 대한 척도로서 자주 쓰이는 것으로 다음과 같이 정의되는 정보행렬(information matrix)을 들 수 있다.

$$
M_{k}(\xi)=\sum_{i=1}^{s} \xi\left(x_{i}\right) f\left(x_{i}\right) f^{T}\left(x_{i}\right)
$$

여기서는 정보행렬이 비특이(nonsingular)인 실험계획에 대해서만 관심을 두고자 한다. 실험계획은 $\left(\Omega, f_{k}(x)\right)$ 과 함께 실험자의 관심을 반영하는 볼록(convex) 실험기준 함수를 결정하여야 하는데 가장 많이 알려진 기준으로서는 $\operatorname{Kiefer(1974)ㅇㅢ~} \Phi_{A P^{-}}$실험기준을 들 수 있다. 만약 $\boldsymbol{A}$ 가 계급 (rank)이 $k+1$ 인 단위행렬 $I$ 이면 이는 $\Phi_{P}\left(M_{k}(\xi)\right)=\left[1 /(k+1) \operatorname{tr}\left(M_{k}^{-P}(\xi)\right]^{1 / P}\right.$ 로 표현되며 이 를 $\Phi_{P^{-}}$실험기준이라 부른다. 특히, $\Phi_{0}, \Phi_{1}$, 그리고 $\Phi_{\infty}$ 의 경우를 $D^{-}, A^{-}$, 그리고 $E^{-}$최적기 준이라 부른다. 간단히 언급하면 $D$-최적은 행렬식 $\left|M_{k}(\xi)\right|$ 을 최대화하는 기준이며 $A$-최적은 $M_{k}^{-1}(\xi)$ 의 트레이스(trace)를 최소화하는 기준이며 $E$-최적은 $M_{k}^{-1}(\xi)$ 의 최대 고유값(eigenvalue) 을 최소화하는 기조이다. 이러한 기준은 $A \theta$ 의 추정이 주 관심사일 때에 적용될 수 있는 기준들 이다. 그리고 Kiefer의 $\Phi_{A P}$ 의 범주 내에는 포함되어 있지 않으나 $y$ 예측치의 분산으로 해석되는 $d_{k}(x, \xi)=f_{k}^{T}(x) M_{k}^{-1}(\xi) f_{k}(x)$ 의 최대값을 최소화하는 $G^{-}$최적도 많이 이용된다. $G^{-}$최적은 $D^{-}$ 최적과 동격임이 잘 알려져 있다. 이러한 기준들은 주어진 모형과 그에 따른 가정이 적절치 못한 경우는 사용에 주의하여야 한다.

모형 (1.1)을 다음과 같이 분해하여 쓰도록 하자.

$$
y=f_{k}^{T}(x) \theta+\varepsilon=f_{j}^{T}(x) \theta_{j}+\sum_{i=j+1}^{k} \theta_{i} x^{i}+\varepsilon
$$

이 경우 $f_{j}(x)$ 모형이 참일 수도 있고 $f_{k}(x)$ 모형이 참일 수도 있다. 실험자는 $f_{j}(x)$ 에 대한 가정을 기본적으로 하여 $\theta_{j}$ 에 대한 정확한 추정에 관심을 기울인다 하더라도 좀 더 포괄적인 모 형이 참일 가능성에 대비해 확장된 모형인 $f_{k}(x)$ 을 사용한다. 왜냐하면 적합결여검정을 실시하는 경우를 염두에 두기 때문이다. 같은 $D$-최적 실험기준을 적용한다 하더라도 모형에 따라 실험이 달라지기 때문에 이 두 모형에 대한 균형을 맞추어야 할 것이다. 또한 모형은 정해져 있다 하더라 도 사용하고자 하는 최적기준은 다양하게 설정될 수 있다. 예를 들어 식 (1.2)에서 $f_{k}(x)$ 에 관련 된 전반적인 모수 $\theta$ 의 추정 뿐 아니라 실험자가 $x^{k}$ 에 관련된 모수 $\theta_{k}$ 의 추정에도 관심을 가질 수 있다. 이때 필요한 실험기준은 $D$-최적과 부분적인 모수 추정과 연관되어 있는 $D_{1}$-최적이다. 여기서 $D_{1}$-최적은 $M_{k}^{-1}(\xi)$ 의 $\theta_{k}$ 에 해당하는 원소 즉, $e_{k}^{T} M_{k}^{-1}(\xi) e_{k}, e_{k}=(0, \cdots, 0,1)^{T}$ 을 최소 화하는 최적기준을 의미한다. Stigler(1971)가 언급한 $C$-제한(restricted) $D$-최적실험은 이를 바 탕으로한 제약최적실험이다.

실험기준들에 우선 순위가 부여될 수 있다고 보자. 그렇다면 주가 되는 실험기준(들)의 효율성 (efficiency)은 어느 정도 보장하면서 부차적인 실험기준의 효율성을 최대화하는 실험기준을 생각 
할 수 있을 것이다. 효율성에 대한 정의는 다음절에서 한다. 이러한 개념을 바탕으로 태어난 것이 제약실험계획법이다. 제약실험계획은 Stigler(1971), Lee(1987), Cook과 Fedorov(1995) 등에 의해 연 구가 진행되었다. 그리고 1994년 Cook과 Wong에 의해 이 방법은 Lauter(1974)의 복합실험계획법 (compound experimental design)과 동격성임이 증명되었다. 여기서 복합실험계획법이란 여러 개 의 경쟁적인 실험기준의 가중평균을 구함으로서 하나의 실험기준을 만드는 방법을 일컫는다. Cook과 Wong(1994)에 의하면 복합실험계획은 적절한 제약조건이 형성이 되어 있으면 대략적으로 제약실험계획의 한 해(solution)로 구현된다. 그러나 이런 결과는 이론적인 면에서 보면 훌륭하나 실제적으로 제약실험계획을 염두에 둔 실험자의 입장에서 보면 많은 어려움을 가져다준다. 두 개 만의 제약조건을 가지고 그 하나는 부차적인 실험기준, 그리고 다른 하나는 주가 되는 실험기준으 로 나누어 설명한 제약실험계획법인 경우는 별 문제가 없으나, 제약조건이 2 개 이상으로 늘어나는 경우는 모든 제약조건을 만족할 만한 해가 존재하는지를 파악할 수 있는 조건식을 찾는 것은 불 가능하기 때문이다. 다음 2절에서는 이로 인한 문제점을 살펴보고 해결할 수 있는 실제적 (practical)인 방안을 살펴보도록 한다. 3절에서는 다항회귀모형을 중심으로 예제를 살펴 본 다음 4 절에서는 실제 실험자의 관점에서 본 논문에서 적용된 방법의 할용에 대해 알아보고자 한다.

\section{2. 다중제약 실험계획법}

어느 기준에 대해 한 실험 $\xi$ 이 최적실험 $\xi^{*}$ 에 대해서 가질 수 있는 효율성을 $\phi_{\xi}\left(\xi^{*}\right)$ 라 한다. 위에서 언급한 여러 실험기준에 대한 효율성은 다음과 같다. 이러한 효율성은 0 과 1 의 범위에서 값을 가진다.

(1) $D$-효율성: $\phi_{\xi}\left(\xi_{k}^{D}\right)=\left\{\left|M_{k}^{-1}\left(\xi_{k}^{D}\right)\right| \mid\left(M_{k}(\xi) \mid\right\}^{1 /(k+1)}\right.$.

여기서 $\xi_{k}^{D}$ 는 차수가 $k$ 인 모형에 대한 $D$-최적실험이다.

(2) $A$-효율성: $\phi_{\xi}\left(\xi_{k}^{A}\right)=\operatorname{tr} M^{-1}\left(\xi_{k}^{A}\right) / \operatorname{tr} M^{-1}(\xi)$.

(3) $G$-효율성: $\phi_{\xi}\left(\xi_{k}^{G}\right)=(k+1) / \max _{x \in \Omega} d(x, \xi)$.

(4) $E$-효율성: $\phi_{\xi}\left(\xi_{k}^{E}\right)=\lambda_{\max }\left(M_{k}^{-1}\left(\xi_{k}^{E}\right)\right) / \lambda_{\max }\left(M_{k}^{-1}(\xi)\right)$.

(5) $D_{1}$-효율성: $\phi_{\xi_{k}}\left(\xi_{k}^{D_{1}}\right)=e_{k}^{T} M_{k}^{-1}\left(\xi_{k}^{D_{1}}\right) e_{k} / e_{k}^{T} M_{k}^{-1}(\xi) e_{k}$. 여기서 $e_{k}=(0, \cdots, 0,1)^{T}$ 이다.

실험기준이 $m$ 개 존재하고 실험이 각각 최적 실험기준 $\xi_{i}^{*}, i=1, \cdots, m$ 에 대해 실험 $\xi$ 가 가지 는 효율성을 $\phi_{\xi}\left(\xi_{i}^{*}\right), i=1, \cdots, m$ 이라 한다면 제약실험계획은 다음과 같이 제약조건하에서의 목적 함수값을 최대화하는 실험 $\xi$ 을 찾는 문제이다.

$$
\max \phi_{\xi}\left(\xi_{l}^{*}\right) \text { subject to } \phi_{\xi}\left(\xi_{i}^{*}\right) \geq c_{i,} i \neq l, i=1, \cdots, m
$$

여기서 $l$ 은 부차적인 목적에 해당되는 실험기준에 해당되는 인덱스이다. 그리고 $c_{i}$ 는 실험자가 설정하여야 하는 0 부터 1 까지의 효율성의 값이다.

그러나 제약조건을 가지는 $m-1$ 개의 실험기준들이 달성하여야 하는 효율성을 실험자가 사전 에 명시하는 것은 그렇게 쉬운 일은 아니다. 이론적인 해를 구하는 것은 거의 불가능하가 때문에 
많은 경우 수치해석적인 알고리즘을 적용하여 구하여야 하는데 이 또한 대부분 소수의 제약조건 하에서만 해를 찾을 수 있다. 왜냐하면 제약조건식이 2 개 이상으로 늘어나는 경우는 모든 제약조 건을 다 만족하는 실험방법이 경우에 따라서 존재하지 않는 경우가 있어 시작점(starting design) 을 찾는 문제가 대두되기 때문이다. 더욱이 Pukelsheim(1993)에 의하면 제약조건이 2개 이상인 경 우 제약최적실험에 대한 구체적인 해(explicit solution)가 일반적으로 존재하지 않는다. 따라서 본 논문에서는 이러한 문제점을 해결할 수 있는 방법을 모색하고자 한다. 본 논문에서는 위에서 언급 한 다항회귀모형을 염두에 둔다.

다항모형에 대한 차수 $k$ 에 대한 확신이 없는 경우 Kussmaul(1969)은 가정하고 있는 차수보다 높은 차수를 가정한 $D$-최적을 제안하였다. 이는 위에서 언급하였듯이 적합결여검정을 염두에 둔 제안이다.

아래 [표 1]은 Wong(1994)이 특정 실험기준의 강건성(robustness)을 확인하기 위해 계산한 표 이다. 본 논문에서 제기하고 있는 절차를 설명하기 위해서는 이에 대한 언급이 필요하여 전재하였

[표 1] $A, D, E, G$-최적의 효율성: $1 \leq j \leq k \leq 8$

\begin{tabular}{|c|c|c|c|c|c|c|c|c|c|}
\hline 차수 & 최적종류 & $\mathrm{k}=1$ & $\mathrm{k}=2$ & $\mathrm{k}=3$ & $\mathrm{k}=4$ & $\mathrm{k}=5$ & $\mathrm{k}=6$ & $\mathrm{k}=7$ & $\mathrm{k}=8$ \\
\hline $\mathrm{j}=1$ & $\mathrm{E}$ & 1.000 & 0.400 & 0.440 & 0.434 & 0.430 & 0.428 & 0.426 & 0.424 \\
\hline & $\mathrm{A}$ & 1.000 & 0.667 & 0.621 & 0.610 & 0.604 & 0.601 & 0.598 & 0.597 \\
\hline & $\mathrm{G}$ & 1.000 & 0.800 & 0.750 & 0.727 & 0.714 & 0.706 & 0.700 & 0.696 \\
\hline & $\mathrm{D}$ & 1.000 & 0.817 & 0.775 & 0.756 & 0.745 & 0.739 & 0.734 & 0.730 \\
\hline $\mathrm{j}=2$ & $\mathrm{E}$ & & 1.000 & 0.439 & 0.503 & 0.502 & 0.503 & 0.504 & 0.504 \\
\hline & $\mathrm{A}$ & & 1.000 & 0.640 & 0.612 & 0.611 & 0.611 & 0.610 & 0.610 \\
\hline & $\mathrm{G}$ & & 1.000 & 0.818 & 0.750 & 0.714 & 0.692 & 0.677 & 0.667 \\
\hline & $\mathrm{D}$ & & 1.000 & 0.865 & 0.828 & 0.809 & 0.798 & 0.790 & 0.784 \\
\hline $\mathrm{j}=3$ & $\mathrm{E}$ & & & 1.000 & 0.434 & 0.504 & 0.500 & 0.500 & 0.500 \\
\hline & $\mathrm{A}$ & & & 1.000 & 0.623 & 0.603 & 0.602 & 0.602 & 0.602 \\
\hline & $\mathrm{G}$ & & & 1.000 & 0.842 & 0.769 & 0.727 & 0.700 & 0.681 \\
\hline & $\mathrm{D}$ & & & 1.000 & 0.895 & 0.863 & 0.847 & 0.836 & 0.829 \\
\hline $\mathrm{j}=4$ & $\mathrm{E}$ & & & & 1.000 & 0.430 & 0.505 & 0.500 & 0.500 \\
\hline & $\mathrm{A}$ & & & & 1.000 & 0.614 & 0.597 & 0.597 & 0.597 \\
\hline & $\mathrm{G}$ & & & & 1.000 & 0.862 & 0.790 & 0.745 & 0.714 \\
\hline & $\mathrm{D}$ & & & & 1.000 & 0.915 & 0.888 & 0.873 & 0.863 \\
\hline $\mathrm{i}=5$ & $\mathrm{E}$ & & & & & 1.000 & 0.428 & 0.506 & 0.500 \\
\hline & $\mathrm{A}$ & & & & & 1.000 & 0.609 & 0.594 & 0.594 \\
\hline & $\mathrm{G}$ & & & & & 1.000 & 0.878 & 0.808 & 0.762 \\
\hline & $\mathrm{D}$ & & & & & 1.000 & 0.928 & 0.904 & 0.891 \\
\hline $\mathrm{i}=6$ & $\mathrm{E}$ & & & & & & 1.000 & 0.426 & 0.507 \\
\hline & $\mathrm{A}$ & & & & & & 1.000 & 0.608 & 0.593 \\
\hline & $\mathrm{G}$ & & & & & & 1.000 & 0.891 & 0.824 \\
\hline & $\mathrm{D}$ & & & & & & 1.000 & 0.945 & 0.917 \\
\hline $\mathrm{j}=7$ & $\mathrm{E}$ & & & & & & & 1.000 & 0.424 \\
\hline & $\mathrm{A}$ & & & & & & & 1.000 & 0.601 \\
\hline & $\mathrm{G}$ & & & & & & & 1.000 & 0.901 \\
\hline & $\mathrm{D}$ & & & & & & & 1.000 & 0.945 \\
\hline
\end{tabular}


다. [표 1]은 가정한 다항모형의 차수 $k$ 가 8 혹은 미만이고 참의 모형이 이보다 낮은 차수 $j$ 로 밝혀졌을 때 나타나는 실험기준에 대한 효율성을 나열하였다. 참의 모형의 차수가 $j$ 로 밝혀졌을 때 가정하였던 모형의 차수 $k$ 가 높아질수록 $A^{-}, G^{-}$, 그리고 $D^{-}$최적인 경우 효율성은 단순감 소(monotonic decreasing)하고 있음을 확인할 수 있다. 그러나 $E$-최적의 경우는 이러한 성질을 부여할 수 없고 그 효율성 또한 매우 낮은 값을 형성하고 있다. 따라서 모형에 대한 확신이 없는 경우 $E$-최적의 사용은 매우 주의를 하여야 한다. 반면 $D$-와 $G$-최적은 상대적으로 높은 효율 성을 유지하고 있음을 알 수 있다.

[표 1]은 매우 홍미로운 결과를 포함하고 있는데 차수를 $k$ 로 가정한 모형에서 나오는 최적실험 $\xi_{k}^{*}$ 이 $j$ 를 차수로 하는 모형에 대해 가지는 효율성은 $D^{-}, G^{-}, A-$, 그리고 $E$-최적의 순에 의 해 다음과 같은 부등식이 성립됨을 알 수 있다.

$$
\phi_{\xi_{k}^{D}}\left(\xi_{j}^{D}\right) \geq \phi_{\xi_{k}^{G}}\left(\xi_{j}^{G}\right) \geq \phi_{\xi_{k}^{A}}\left(\xi_{j}^{A}\right) \geq \phi_{\xi_{k}^{E}}\left(\xi_{j}^{E}\right)
$$

위에서 언급한 바와 같이 $D-, A-$, 그리고 $E$-최적은 각각 Kiefer의 $\Phi_{A P}$ 에 $p=0,1$ 그리고 $\infty$ 에 해당된다고 본 바 있다. 따라서 $G$-최적은 이 범주에 속하지 않지만 0 과 1 사이에 존재한 다고 유추해 볼 수 있을 것이다. Atwood(1969)는 $D$-최적과 $G$-최적의 부등식을 일반적인 $\xi$ 에 대해서도 정리한 바 있다.

[표 1]을 본 논문의 관점에서 살펴보자. 실험자가 선택하고자 하는 차수가 $k=1$ 또는 $k=2$ 라 가정하자. $f_{1}$ 모형에 대한 효율성을 주가 되는 제약조건이라 한다면 식 (2.1)에서 정의한 $c_{1}$ 값이 $81.7 \%$ 이하로 설정되어 있는 제약 $D$-최적실험계획은 $f_{2}$ 를 염두에 둔 $D$-최적인 $\xi_{2}^{D}( \pm 1)=\xi_{2}^{D}(0)$ $=1 / 3$ 이 된다. 그렇지 않고 $c_{1}$ 값이 $81.7 \%$ 이상으로 효율성이 정해졌으면 제약실험계획은 각 받침 점에 대한 질량을 달리 할 것이다. Lee(1987)의 논문은 이러한 내용을 바탕으로 한 것이다. 그러 나 Lee(1987)는 한 개의 제약조건만을 명기하였기 때문에 3 개 이상의 다목적 실험기준을 가지고 있는 실험자에게는 많은 도움을 주지 못한다.

이제 3 개의 제약조건이 있는 경우를 생각하여 보자. 실험자가 가정하고 있는 차수는 $k=4$ 이다. 그러나 참의 모형은 $f_{1}$ 이 될지 $f_{2}$ 이 될지 아니면 $f_{3}$ 모형이 될지 모르는 상황이다. 따라서 실험의 효율성을 일차인 경우 $75.6 \%$ 와 이차인 경우 $82.8 \%$ 그리고 3 차인 경우 $89.5 \%$ 이하로 설정한다면 $\xi_{4}^{D}$ 이 제약 $D$-최적실험계획이 될 것이다. 그러나 세 개의 제약조건중 하나라도 효율성의 하한값 이 이보다 높게 설정되면 최적실험계획을 구하는 것은 매우 어려운 작업이 된다. 경우에 따라서는 타당치 않은(infeasible) 해가 나올 가능성이 있으며 설사 타당한(feasible) 해가 존재한다 하더라도 해를 구하는 작업은 제약조건식의 개수가 많아질수록 많은 어려움이 따를 것이다. 따라서 본 논문 은 다음과 같은 방법을 제시한다.

(1) 실험자가 $m$ 개의 실험기준을 가지고 있다고 가정한다. 실험자는 실험기준에 대해 우선순위 를 부여할 수 있다. 편의상 $m=1$ 에 해당하는 실험기준을 실험자가 제일 우선하여 만족시켜야 하 는 주 제약조건이라 하자. 즉, 높은 $m$ 값은 우선순위가 낮은 제약조건이다.

(2) 첫 번째 주 제약조건을 만족하는 범위 내에서 두 번째 실험기준의 효율성인 목적함수를 최 
대화하는 실험을 구한다. 구해진 목적함수의 최대값을 $\max \phi_{\xi}\left(\xi_{2}^{*}\right)=c_{2}^{*}$ 로 놓는다. 여기서 $c_{1}$ 은 실험자가 임의로 설정한다.

$$
\max \phi_{\xi}\left(\xi_{2}^{*}\right) \text { subject to } \phi_{\xi}\left(\xi_{1}^{*}\right) \geq c_{1}
$$

(3) 세 번째 실험기준을 목적함수로 하는 두 개의 제약조건식을 설정한다.

$$
\max \phi_{\xi}\left(\xi_{3}^{*}\right) \text { subject to } \phi_{\xi}\left(\xi_{1}^{*}\right) \geq c_{1} \text { and } \phi_{\xi}\left(\xi_{2}^{*}\right) \geq c_{2}
$$

여기서 $c_{2}$ 는 $c_{2}^{*}$ 와 $c_{2}^{*}>c_{2}$ 의 관계를 가지게 함으로서 타당한 해가 존재토록 한다.

(4) 이와 같은 방법으로 모든 제약조건식을 소화한다. 이렇게 구해진 실험을 앞으로 제약최적실 험이라 칭한다.

이런 방법은 타당치 않은 해의 위험을 피해 갈 수 있는 순차적인 알고리즘이기 때문에 항상 시 작점(starting design)이 주어지므로 해를 구하는데 어려움이 없다. 또한 우선순위가 높은 실험기 준이 주 제약조건으로 순차적으로 들어감으로써 실험자가 명시한 효율성을 충족시킬 수 있는 장 점이 있다. 그런 후 다음으로 중요한 부차적인 목적이 $100 \%$ 달성되지 못할 수 있다는 제약성을 염두에 두고 실험을 하는 방법이 일시에 모든 제약조건에 대한 효율성을 부여하는 것보다는 여러 면에서 낫다고 본다. 다음절에서는 이와 같은 내용을 가지고 몇 가지 예제를 제시하여 보고자 한 다. 다음절에 소개되는 예제들에 대한 해는 Mikulecka(1983)을 변형하거나 염준근과 남기성(2000) 의 유전자 알고리즘 방법을 적용하여 구하였다. 알고리즘은 그들의 논문을 참조 바란다.

\section{3. 예 제}

예제 1 실험자는 차수 1,2 , 그리고 3 에 대한 모형의 마지막 회귀계수에 대한 추정에 많은 관심 을 가지고 있다. 단 차수 $1,3,2$ 에 해당되는 $D_{1}$-최적 실험기준이 차례로 우선순위가 부여된다. 두 개의 제약조건에 해당되는 $D_{1}$-효율성 $\phi_{\xi}\left(\xi_{1}^{D_{1}}\right), \phi_{\xi}\left(\xi_{3}^{D_{1}}\right)$ 은 적어도 $c_{i}, i=1,2$ 만큼은 보장하 고자 한다. 그런 다음 $\phi_{\xi}\left(\xi_{2}^{D_{1}}\right)$ 의 최대값을 구하고자 한다. Dette와 Franke(2000)의 논문에 의하면 $c_{1}$ 값이 $1 / 2$ 이하로 설정되는 경우는 제약최적실험의 해는 항상 존재하고 $c_{1}$ 값이 $1 / 2$ 보다 크게 설정되는 경우 $c_{2} \leq 4 c_{1}\left(1-c_{1}\right)$ 를 만족하면 제약최적실험이 존재한다고 하였다. 그러나 일반 실 험자는 이러한 사실을 모르는 바 본 논문에서 제시한 순차적인 제약실험 방법을 통하여 해를 구 하여 볼 수 있을 것이다. 순차적인 실험결과는 다음과 같은 그들의 결과와 일치함을 알 수 있다.

(1) $c_{1} \leq 1 / 2$ 인 경우 각 효율성

$$
\phi_{\xi}\left(\xi_{1}^{D_{1}}\right)=0.5, \quad \phi_{\xi}\left(\xi_{3}^{D_{1}}\right)=c_{2}, \quad \phi_{\xi}\left(\xi_{2}^{D_{1}}\right)=1 / 2\left(1+\sqrt{1-c_{2}}\right)
$$

(2) $c_{1}>1 / 2$ 이고 $c_{2} \leq 4 c_{1}\left(1-c_{1}\right)$ 인 조건식을 만족하는 경우 각 효율성

$$
\phi_{\xi}\left(\xi_{1}^{D_{1}}\right)=c_{1}, \quad \phi_{\xi}\left(\xi_{3}^{D_{1}}\right)=c_{2}, \quad \phi_{\xi}\left(\xi_{2}^{D_{1}}\right)=2 c_{1}\left(1-c_{1}\right)\left(1+\sqrt{1-\frac{c_{2}}{4 c_{1}\left(1-c_{1}\right)}}\right)
$$

그리고 질량은 다음과 같이 부여된다. 


$$
\xi( \pm 1)=\alpha, \quad \xi\left( \pm \sqrt{p_{1}\left(1-p_{2}\right)}\right)=1 / 2-\alpha
$$

여기서 $\alpha=p_{1} p_{2} / 2\left(1-p_{1}\left(1-p_{2}\right)\right)$ 이며 $p_{1}$ 과 $p_{2}$ 는 다음과 같다.

$$
\begin{aligned}
& p_{1}=\max \left[1 / 2, c_{1}\right] \\
& p_{2}=\frac{1}{2}\left(1+\sqrt{1-c_{2}}\right) \quad \text { if } \quad c_{1} \leq \frac{1}{2} \\
& p_{2}=\frac{1}{2}\left(1+\sqrt{1-\frac{c_{2}}{4 c_{1}\left(1-c_{1}\right)}}\right) \text { if } c_{1}>\frac{1}{2}
\end{aligned}
$$

자세한 내용은 그들의 논문을 참조 바란다.

예제 2 실험자가 가정하는 다항회귀모형의 차수는 $k=3$ 이다. 그러나 일차모형이 참으로 밝혀질 것에 대비한 효율성 $\phi_{\xi}\left(\xi_{1}^{D}\right)$ 을 $c_{1}$ 이상으로 설정하고자 한다. 그리고 2 차모형이 참으로 밝혀질 것 에 대비한 효율성 $\phi_{\xi}\left(\xi_{2}^{D}\right)$ 을 $c_{2}$ 이상으로 정하고 싶다. [표 1]에서와 같이 효율성의 하한값 $c_{1}$ 이 $81.7 \%(=\sqrt{2 / 3})$ 보다 높게 설정되면 2 차모형에 대해 실험이 가질 수 있는 효율성 $\phi_{\xi}\left(\xi_{2}^{D}\right)$ 은 $100 \%$ 를 보장하지 못한다. 이 경우 목적함수가 가질 수 있는 최대값 $c_{2}^{*}$ 은 $\left[27(1-\xi(0))^{2} \xi(0) / 4\right]^{1 / 3}$ 로 나타난다. 여기서 $\xi(0)=1-c_{1}^{2}$ 이다. 물론 $c_{1}$ 이 $\sqrt{2 / 3}$ 이하로 설정되는 경우는 $100 \%$ 의 효율성을 보장한다. 따라서 이 문제는 $c_{1}$ 값에 따른 목적함수의 최대 효율성을 계산한 후 이 보다 작은 값 을 $c_{2}$ 로 하여 $\phi_{\xi}\left(\xi_{3}^{D}\right)$ 의 최대값을 구하는 실험이 될 것이다. [표 1]에서 보듯이 $c_{1}$ 값이 0.775 그 리고 $c_{2}$ 값이 0.865 이하로 설정되는 경우는 $\xi_{3}^{D}$ 이 제약최적실험이 될 것이다. 그렇지 않은 경우는 수치적으로 해를 구하여야 한다. Dette와 Franke(2000)의 방법은 너무 복잡하기 때문이다. 아래 두 실험에 대한 해는 수치적으로 구하였다. 첫째 경우는 목적함수의 $c_{2}^{*}$ 값이 1.00 이 나오므로 $c_{2}$ 값은 이보다 작은 값 0.9 를 임의로 선택하였으며 두 번째 경우도 $c_{2}^{*}$ 값이 0.98277 로 나와 이보다 작은 값 0.9 를 임의로 선택하였다. 참고로 $\xi_{3}^{D}$ 은 받침점 $\pm \sqrt{5}, \pm 1$ 에 등질량 $1 / 4$ 을 가진다.

(1) $c_{1}=\sqrt{2 / 3}, \quad c_{2}=0.9$ 인 경우 목적함수의 효율성 $\phi_{\xi}\left(\xi_{3}^{D}\right)$ 는 0.9788 의 값을 가진다. 아래가 제약최적실험결과이다.

$$
\xi( \pm 1)=0.2963, \quad \xi( \pm 0.4325)=0.1980, \quad \xi(0)=0.01138
$$

(2) $c_{1}=\sqrt{3 / 4}, c_{2}=0.9$ 인 경우 $\phi_{\xi}\left(\xi_{3}^{D}\right)$ 는 0.9154 값을 가진다. $f_{1}$ 에 대한 효율성을 높이기 위 한 질량 이동이 이루어 졌음을 알 수 있다.

$$
\xi( \pm 1)=0.3486, \quad \xi( \pm 0.4204)=0.14950, \quad \xi(0)=0.00385
$$

위 두 경우는 제약조건에 해당하는 실험의 효율성 $\phi_{\xi}\left(\xi_{1}^{D}\right), \phi_{\xi}\left(\xi_{2}^{D}\right)$ 은 모두 설정한 하한값과 일치 한다.

(3) [표 1]에 의해 $c_{1}$ 과 $c_{2}$ 값이 각각 $0.775,0.865$ 이하로 설정될 경우는 물론 $\xi_{3}^{D}$ 이 제약최적실 
험이다. 그리고 목적함수는 1.00 을 취한다.

예제 3 실험자가 가정하는 차수는 $k=2$ 이다. $f_{1}$ 이 참의 모형으로 밝혀질 경우에 대비하여 부여 해야할 최적실험의 기준은 하나가 아닌 두 개로 $D$-와 $A$-최적이라고 하자. $A$-효율성 $\phi_{\xi}\left(\xi_{1}^{A}\right)$ 과 $D$-효율성 $\phi_{\xi}\left(\xi_{1}^{D}\right)$ 이 각각 일정수준 이상으로 유지하면서 $f_{2}$ 모형에 대한 $D$-효율성 $\phi_{\xi}\left(\xi_{2}^{D}\right)$ 을 최대화하는 제약최적실험을 구하는 문제이다. 일차모형에 대한 $A$-최적과 $D$-최적은 모두 $\xi( \pm 1)=1 / 2$ 이므로 $c_{1}$ 과 $c_{2}$ 의 값은 1.00 보다 작은 값으로 설정하면 된다. 이렇게 구한 제약최 적실험을 보면 가운데 받침점 0 에 대한 질량 $\xi(0)$ 은 0 보다 크나 $1 / 3$ 보다 작은 값을 가지되 제약 조건식을 위배하지 않는 범위 내에서 정하여진다. 다음 두 경우를 살펴보자.

(1) $c_{1}$ 과 $c_{2}$ 값이 모두 0.85 로 설정될 때 제약최적실험이 아래 주어져 있다. 목적함수의 효율성, $\phi_{\xi}\left(\xi_{2}^{D}\right)$ 은 0.9872 이다. 두 번째 제약조건에 해당되는 $D$-효율성, $\phi_{\xi}\left(\xi_{1}^{D}\right)$ 은 0.8597 로 설정한 하한 값 0.85 보다 높게 나와 초과 달성하였다. 반면 $A$-효율성, $\phi_{\xi}\left(\xi_{1}^{A}\right)$ 은 0.85 이다.

$$
\xi( \pm 1)=0.3696, \xi(0)=0.26087
$$

(2) $\phi_{\xi_{2}^{v}}\left(\xi_{1}^{A}\right)$ 이 0.8 이고 [표 1]에 의해 $\phi_{\xi_{2}^{0}}\left(\xi_{1}^{D}\right)=\sqrt{2 / 3}$ 이므로 $c_{1}$ 과 $c_{2}$ 값이 각각 0.8 과 $\sqrt{(2 / 3)}$ 이하로 설정될 경우는 $\xi_{2}^{D}$ 이 제약최적실험이다. 따라서 목적함수 $\phi_{\xi}\left(\xi_{2}^{D}\right)$ 는 항상 1.00 을 취한다.

\section{4. 결 론}

하나의 기준만을 가지고 실험계획을 세운다는 것은 결코 바람직하지 않다. 가능하면 많은 기준 들을 수용하는 실험계획이 바람직하다. 이러한 문제를 해결하기 위해서 나타난 것의 하나가 제약실 험계획이다. 그러나 제약조건이 2개 이상 설정되는 제약조건실험인 경우는 각 제약조건식의 효율 성을 설정하는 문 제가 대두된다. Dette와 Franke(2000)이 제시한 Canonical Moments을 이용한 방 법은 일부 예제에 대해서만 정확(exact)한 해를 구할 뿐이다. 대부분의 경우는 본 논문에서 제시 한 제약조건의 우선순위를 부여하여 순차적으로 타당한 해를 찾아가는 방법을 이용하여 제약조건 실험에 대한 최적해를 구하여야 한다. 단 제약조건의 하한값을 각 단계별로 적절하게 설정하여야 하는 논제가 대두될 수 있다. 그러나 실험자가 생각하는 제약조건에 명시되는 효율성의 값은 다항 회귀모형인 경우는 [표 1]을 참조하면 많은 도움을 받을 수 있다고 본다. 3절에서는 Lee(1987)와 Cook과 Wong(1994)의 논문에 나온 예제들을 중심으로 본 논문에서 제시된 방법을 설명하였다.

\section{참고문헌}

[1] 염준근, 남기성 (2000). A study on $D$-optimal design using the genetic algorithm, $\ulcorner$ 한국 통계학회논문집」, 제7권 1호, 357-366.

[2] Atwood, C. L. (1969). Optimal and efficient designs of experiments, Annals of Mathematical Statistics. Vol. 40. 1570-1602. 
[3] Cook, R. D. and Wong, W. K. (1994), On the equivalence between constrained and compound optimal designs, Journal of the American Statistical Association, Vol. 89, 687-692.

[4] Cook, R. D. and Fedorov, V. V. (1995). Constrained optimization of experimental design with discussion, Statistics, Vol. 26, 129-178.

[5] Dette, H, and Franke, T. (2000). Constrained $D_{1}$ - and $D$-optimal designs for polynomial regression, Ruhr-Universitat Bochum technical paper.

[6] Kiefer, J. (1974). General equivalence theory for optimal designs (approximate theory), Annals of Statistics, Vol. 2, 849-879

[7] Kussmaul, K. (1969). Protection against assuming the wrong degree in polynomial regression, Technometrics, Vol. 11, 677-682.

[8] Lauter, E. (1974). Experimental planning in a class of models, Mathematishe Operationsforshung und Statistik, Vol. 5, 673-708.

[9] Lee, C. M. S. (1987). Constrained optimal designs for regression models, Communications in Statistics, Part A-theory and Methods, Vol. 16, 765-783.

[10] Mikulecka, J. (1983). On hybrid experimental design, Kybernetika, Vol. 19, 1-14.

[11] Pukelsheim, F. (1993). Optimal Design of Experiments, Wiley, New York.

[12] Stigler, S. M. (1971). Optimal experimental design for polynomial regression, Journal of the American Statistical Association, Vol. 66, 311-318.

[13] Wong, W. K. (1994). Comparing robust properties of $A, D, E$ and $G$-optimal designs, Computational Statistics \& Data Analysis, Vol. 18, 441-448. 